\title{
La resolución de problemas con distintos actores: una actividad basada en la economía colaborativa
}

\section{María de-Miguel-Molina ${ }^{a}$ y Daniel Catalá-Pérez}

${ }^{a}$ Departamento de Organización de Empresas, Universitat Politècnica de València, mademi@omp.upv.es, 'bepartamento de Organización de Empresas, Universitat Politècnica de València, dacape@omp.upv.es

\begin{abstract}
The collaborative economy presents new business models through digital platforms that sometimes fight with services that have traditionally been provided with strong regulation. This may be the case of the taxi sector, which requires to be reformulated in order to be competitive. In this activity, we analyze the need to raise these issues through a governance model where all stakeholders can express their opinions, trying to achieve solutions as consensual as possible. This requires an analysis of the regulations involved and the opinions of the users, without losing sight of the economic groups in conflict. In order to achieve this consensus, the public administration will play a crucial role in the future.
\end{abstract}

Keywords: collaborative economy, stakeholders, transport, regulation, public administration, public-private partnerships.

\begin{abstract}
Resumen
La economía colaborativa presenta nuevos modelos de negocio a través de plataformas digitales que, en ocasiones, chocan con servicios que tradicionalmente se han prestado con una fuerte regulación. Este puede ser el caso del sector del taxi, que precisa de una reformulación para poder ser competitivo. En esta actividad, se analiza la necesidad de plantear estos temas a través de un modelo de gobernanza donde todos los grupos de interés puedan expresar su opinión y se intenten conseguir soluciones lo más consensuadas posible. Ello requiere un análisis de la normativa implicada y de la opinión de los usuarios, sin perder de vista los grupos económicos en conflicto. Para lograr este consenso, el papel que juegue la administración pública en el futuro será fundamental.
\end{abstract}

Palabras clave: economía colaborativa, grupos de interés, transporte, regulación, modelos de colaboración público-privada. 


\section{Introducción}

De acuerdo con la Comisión Europea (2016: 3), la economía colaborativa se basa en,

"modelos de negocio en los que se facilitan actividades mediante plataformas colaborativas que crean un mercado abierto para el uso temporal de mercancías o servicios ofrecidos a menudo por particulares. La economía colaborativa implica a tres categorías de agentes i) prestadores de servicios que comparten activos, recursos, tiempo y/o competencias pueden ser particulares que ofrecen servicios de manera ocasional («pares») o prestadores de servicios que actúen a título profesional («prestadores de servicios profesionales»); ii) usuarios de dichos servicios; y iii) intermediarios que -a través de una plataforma en línea - conectan a los prestadores con los usuarios y facilitan las transacciones entre ellos («plataformas colaborativas»). Por lo general, las transacciones de la economía colaborativa no implican un cambio de propiedad y pueden realizarse con o sin ánimo de lucro".

A pesar de esta extensa explicación, el concepto no cuenta con la unanimidad académica y determinados servicios se consideran o no "economía colaborativa" en función del autor y de las distintas interpretaciones que han realizado los tribunales.

Para Doménech (2015: 66),

"La causa principal del surgimiento de la economía colaborativa es.. el espectacular progreso experimentado por las tecnologías de la información, que ha posibilitado abaratar exponencialmente las actividades de obtención, almacenamiento, procesamiento y comunicación de información que los interesados han de llevar a cabo para colaborar en la producción y consumo de bienes y servicios, intercambiándolos o compartiéndolos”.

Dentro de la economía colaborativa, el sector del transporte, y concretamente el transporte público, se ha visto salpicado por distintas interpretaciones, normativas, e incluso conflictos debido al impacto de la economía digital. Y, específicamente, el transporte público de taxis en España ha sido objeto de fuertes disputas.

Servicios de transporte de pasajeros, como Uber o Cabify, han tenido un impacto distinto en función del Estado Europeo en el que nos encontremos y, dentro de España, en cada comunidad autónoma. Incluso, más allá de estos conflictos, nos podemos preguntar si este caso entraría o no dentro de la economía colaborativa.

Más aún, el Tribunal de Justicia de la Unión Europea, en sentencia de 20 de diciembre de 2017, en el asunto de la Asociación Profesional Élite Taxi contra Uber Systems Spain, S.L. (TSJUE, 2017), falló en contra de Uber, indicando que no era un servicio de intermediación ni una libre prestación de servicios y cualquiera no puede darse de alta.

Por ello, siendo tantos los grupos de interés implicados en ello, es un caso de análisis muy interesante para nuestros alumnos del Grado en Gestión y Administración Pública. Sin embargo, y según el punto de vista que tomemos, puede ser también de interés para otros Grados como Administración y Dirección de Empresas o Ingeniería Informática.

La economía colaborativa, fundamentalmente, reformula modelos de negocio que tradicionalmente hemos conocido de una manera, pero que el futuro les lleva a tener que

(cc) EY-NC-ND 2018, Universitat Politècnica de València

Congreso IN-RED (2018) 
reinventarse y replantearse cómo afrontar estos cambios. Blablacar, por ejemlo, es otro modelo sustitutivo completamente distinto, pero con el mismo objetivo. Y siendo un transporte público, el papel que juegue la administración pública también es fundamental.

\section{Objetivos}

La práctica, titulada "La guerra de los taxis", se planteó dentro del aula para trabajar en grupo, y se encuadró dentro del tema "La colaboración público-privada”, de la asignatura Dirección Estratégica de Organizaciones Públicas (Catalá Pérez, 2017), que se imparte en el $3^{\circ}$ curso del Grado en Gestión y Administración Pública.

El objetivo de la dinámica fue analizar un problema complejo y buscar soluciones de colaboración público-privada utilizando los mapas conceptuales móviles (De Miguel et al, 2017a), de manera que planteasen una solución en equipo. De este modo, se relacionaba este tema con otros dados en la asignatura como el trabajo en equipo o la gobernanza (De Miguel et al, 2017b), puesto que el conseguir el mayor consenso posible entre los grupos de interés cada vez resulta más necesario para dar legitimidad a las decisiones públicas.

Las tareas a desarrollar fueron cuatro:

1. Identificar problemas (el reto): ¿es posible un sistema de colaboración público-privada para el servicio de taxis? Ventajas e inconvenientes (¿para quién? Identificar los distintos actores).

2. Brainstorming de ideas: ¿qué soluciones de colaboración público-privada serían posibles? Votar las mejores para reducir las soluciones a máximo 3.

3. Captar nuevas ideas: los grupos van rotando para explicar sus soluciones y que los demás les den feedback.

4. Selección y revisión de una única solución de colaboración público-privada.

\section{Desarrollo de la innovación}

Para identificar los problemas, se les proporcionó a los alumnos diversas noticias extraídas de la prensa para analizar la situación:

- Conflictos con las licencias VTC (para arrendamiento de vehículos de turismo con conductor) para chóferes privados, pues su número ha ido variando con el tiempo lo que ha provocado paralizaciones de solicitudes y sentencias que, en ocasiones, han obligado a otorgarlas durante unos años (Fernández, 2017).

- Empresas que han planteado alternativas al taxi, como Uber o Cabify (Otero, 2017).

- Diferentes interpretaciones sobre lo que se considera "liberalizar el mercado" (Domínguez del Valle, 2017 y Herce, 2017).

- Nuevas demandas por parte de los usuarios (Esteve, 2016).

(cc)) EY-NG-ND 2018, Universitat Politècnica de València

Congreso In-Red (2018) 
- Regulación del taxi en España basada en un modelo de licencias muy caras frente a las VTC, traspaso o acumulación de licencias, grupos de taxistas divididos aunque han conseguido crear una app conjunta (MyTaxi, 2016).

Después de este análisis por equipos de 3-5 alumnos, los profesores repartieron unas tarjetas post-it de distintos tamaños, colores y formas para desarrollar cada punto de la práctica. En este caso, necesitaron 4 tipos diferentes.

1. Tras haber identificado los distintos problemas, los podían expresar con palabras clave o dibujos en notas móviles de un color determinado (en este caso naranja, Imagen 1). Era importante ponerse en la "piel" de los distintos actores para tomar perspectiva. En este sentido, también se analizaron las ventajas de cada servicio (en este caso rosa, Imagen 1).

2. De acuerdo con el proceso de resolución de problemas, los grupos buscaron soluciones de colaboración público-privada para cada problema por medio de la tormenta de ideas (brainstorming), de forma que se seleccioban como máximo 3 soluciones consensuadas. Dichas soluciones se expresaban también con palabras clave o dibujos en una nota móvil de otro color (en este caso azul, Imagen 1), y se conectaron con los problemas a resolver. En este momento, el profesor revisó los modelos de cada grupo para detectar información que faltase por completar. Al mostrar este modelo con las notas móviles pegadas en la pared, teníamos tres filas de ideas: problemas, ventajas y soluciones.

3. Con estas posibilidades, el resto de grupos se desplazaron por el aula para observar los modelos del resto de equipos y así poder obtener feedback de los compañeros. La mitad del grupo se quedaba en su sitio para explicar dudas a los "visitantes" o recoger las "recomendaciones" de sus compañeros. Acabada la ronda, cada grupo volvía a reunirse para enriquecer un modelo específico de colaboración público-privada con todas las ideas aportadas (Tabla 1). Se eligía y revisaba el que pensaban que podía cubrir mejor las "necesidades” de los "actores” que habían dado su feeback. Esta última solución se mostró en una cuarta fila con otro tipo de nota móvil (en este caso, más grande y de color verde, Imagen 1).

Tabla 1. Tipos de colaboración público-privada contractual

\begin{tabular}{|l|l|l|}
\hline TIPO & $\begin{array}{l}\text { TAREAS } \\
\text { OPERACIONES }\end{array}$ & DESCRIPCIÓN \\
\hline O\&M & Operate-Mantain & $\begin{array}{l}\text { El sector público contrata con un agente privado la gestión } \\
\text { y mantenimiento de un servicio específico. El sector } \\
\text { público retiene propiedad y gestión general de la instalación } \\
\text { o sistema público. }\end{array}$ \\
\hline DB & $\begin{array}{l}\text { Lesign-Build } \\
\text { La más básica de las fórmulas de CPP y la que menos } \\
\text { obligaciones y riesgos transfiere al sector privado. El agente } \\
\text { privado diseña y construye el proyecto. El organismo } \\
\text { público es responsable de la financiación, pero ahorra } \\
\text { costos y tiempo de realizarlo en contratos separados y } \\
\text { puede formalizar una operación de O\&M con el agente }\end{array}$ \\
\hline
\end{tabular}

(cc) EY-NC-ND 2018, Universitat Politècnica de València 


\begin{tabular}{|c|c|c|}
\hline & & privado u realizar estas tareas de forma interna. \\
\hline DBO & $\begin{array}{l}\text { Design-Build- } \\
\text { Operate }\end{array}$ & $\begin{array}{l}\text { Similar a la anterior excepto por que el agente privado } \\
\text { también gestiona el proyecto. La gestión de proyectos a } \\
\text { gran escala habitualmente requiere de una amplia } \\
\text { experiencia técnica y de importantes inversiones en } \\
\text { personal. Esta fórmula permite al sector público transferir } \\
\text { esta responsabilidad al agente privado, mientras mantiene } \\
\text { las de financiación de la construcción y del mantenimiento. }\end{array}$ \\
\hline DBM & $\begin{array}{l}\text { Design-Build- } \\
\text { Mantain }\end{array}$ & $\begin{array}{l}\text { Similar a la estructura DB, excepto en que el sector privado } \\
\text { también mantiene el proyecto. El sector público paga una } \\
\text { cantidad acordada por estos servicios y si se requieren más } \\
\text { fondos, suele ser responsabilidad del sector privado } \\
\text { asumirlos. Ser capaz de trasladar la responsabilidad de las } \\
\text { reparaciones al agente privado puede resultar en ahorros } \\
\text { significativos para el sector público. }\end{array}$ \\
\hline DBOM & $\begin{array}{l}\text { Design-Build- } \\
\text { Operate-Maintain }\end{array}$ & $\begin{array}{l}\text { El agente privado es responsable del diseño, construcción y } \\
\text { de la O\&M del proyecto. El sector público mantiene la } \\
\text { propiedad y es responsable de la financiación de la } \\
\text { construcción y obtiene la ventaja de transferir al agente } \\
\text { privado la responsabilidad sobre la gestión y el } \\
\text { mantenimiento. }\end{array}$ \\
\hline DBF & $\begin{array}{l}\text { Design-Build- } \\
\text { Finance }\end{array}$ & $\begin{array}{l}\text { Con este se adjudica un contrato por el diseño, la } \\
\text { construcción y la financiación total o parcial de una } \\
\text { instalación. La responsabilidad del mantenimiento a largo } \\
\text { plazo y la explotación de la instalación permanecen en el } \\
\text { sector público. Este enfoque permite al sector público } \\
\text { aplazar la financiación durante la fase de construcción del } \\
\text { proyecto. }\end{array}$ \\
\hline DBFO & $\begin{array}{l}\text { Design-Build- } \\
\text { Finance-Operate }\end{array}$ & $\begin{array}{l}\text { Esta estructura es similar a la estructura de DBO, excepto } \\
\text { que el agente privado también es responsable de la } \\
\text { financiación del proyecto. El agente privado puede cobrar } \\
\text { del organismo público o de los usuarios finales del } \\
\text { proyecto. }\end{array}$ \\
\hline DBFOM & $\begin{array}{l}\text { Design-Build- } \\
\text { Finance-Operate- } \\
\text { Maintain }\end{array}$ & $\begin{array}{l}\text { Bajo esta estructura, el agente privado es responsable del } \\
\text { diseño, construcción, financiación, gestión y mantenimiento } \\
\text { del proyecto durante un período especificado. En esta } \\
\text { estructura, el proyecto es propiedad del sector público. El } \\
\text { agente privado puede cobrar del organismo público o de los } \\
\text { usuarios finales del proyecto. }\end{array}$ \\
\hline DBFOMT & $\begin{array}{l}\text { Design-Build- } \\
\text { Finance-Operate- } \\
\text { Maintain- Transfer }\end{array}$ & $\begin{array}{l}\text { Esta estructura es similar al modelo DBFOM, excepto que } \\
\text { el sector privado posee el activo durante el plazo del } \\
\text { contrato de PPP después de lo cual la propiedad, operación } \\
\text { y mantenimiento son transferidos a la agencia pública. }\end{array}$ \\
\hline BOT & Build-Operate- & Un consorcio privado recibe licencia de la Administración \\
\hline
\end{tabular}




\begin{tabular}{|c|c|c|}
\hline & Transfer & $\begin{array}{l}\text { para financiar y construir un equipamiento, otorgándole al } \\
\text { mismo tiempo la concesión para explotarlo comercialmente } \\
\text { durante un determinado período. Finalizado el plazo de } \\
\text { concesión, la instalación pasará a depender de la } \\
\text { Administración Pública, quien deberá decidir si se encarga } \\
\text { directamente de su explotación o la cede a un operador } \\
\text { privado. El plazo de concesión se intenta establecer a priori } \\
\text { de forma que la entidad privada haya obtenido una } \\
\text { rentabilidad adecuada sobre la inversión inicial realizada. }\end{array}$ \\
\hline BTO & $\begin{array}{l}\text { Build-Transfer- } \\
\text { Operate }\end{array}$ & $\begin{array}{l}\text { Se trata de un sistema similar al anterior, pero en esta } \\
\text { ocasión las instalaciones para la prestación del servicio } \\
\text { pasan a ser propiedad de la Administración contratante, } \\
\text { inmediatamente, tras finalizar su construcción por el } \\
\text { operador privado. Éste tendrá el derecho a explotarlas } \\
\text { durante un cierto período, en virtud de un acuerdo de } \\
\text { arrendamiento a largo plazo que deberá concederle la } \\
\text { Administración Pública, al final del cual la Administración } \\
\text { debe decidir entre explotar el proyecto directamente o } \\
\text { volver a arrendarlo a un operador privado, que ahora no } \\
\text { tiene que ser la entidad que inicialmente lo desarrolló. }\end{array}$ \\
\hline BROT & $\begin{array}{l}\text { Build-Rent-Operate- } \\
\text { Transfer }\end{array}$ & $\begin{array}{l}\text { Se trata de una variante de los proyectos BOT ó BTO, en la } \\
\text { que el operador privado explota las instalaciones pero } \\
\text { arrienda los bienes físicos por la duración del acuerdo. }\end{array}$ \\
\hline BLOT & $\begin{array}{l}\text { Build-Lease- } \\
\text { Operate-Transfer }\end{array}$ & $\begin{array}{l}\text { Variedad del anterior método en la que en el arrendamiento } \\
\text { se contempla la posibilidad de que el arrendatario ejercite } \\
\text { una opción de compra. }\end{array}$ \\
\hline BOOT & $\begin{array}{l}\text { Build-Own-Operate- } \\
\text { Transfer }\end{array}$ & $\begin{array}{l}\text { En este caso, el operador privado se compromete a } \\
\text { financiar, construir, explotar y mantener una infraestructura, } \\
\text { a cambio del derecho a cobrar por ello a los usuarios. En } \\
\text { virtud de este acuerdo la entidad privada es propietaria de } \\
\text { las instalaciones y los bienes hasta el final de la concesión. } \\
\text { En cualquier caso, puede estar sujeta a restricciones legales } \\
\text { sobre su gestión o las tarifas a cobrar. }\end{array}$ \\
\hline BOO & Build-Own-Operate & $\begin{array}{l}\text { Variación de la fórmula BOOT; el operador privado es } \\
\text { propietario perpetuo del equipamiento y no tiene obligación } \\
\text { de traspasarlo a la Administración pero tiene la opción de } \\
\text { comprarlo. }\end{array}$ \\
\hline BBO & Buy-Build-Operate & $\begin{array}{l}\text { Un operador privado adquiere un activo a la Administración } \\
\text { Pública. Aquél se ocupa de la renovación o ampliación } \\
\text { necesarias del equipamiento para la prestación de un } \\
\text { servicio público, según la demanda, sin que se establezca un } \\
\text { momento de finalización. La entidad pública puede utilizar } \\
\text { el acuerdo de licencia para la prestación del servicio para } \\
\text { establecer las condiciones de acceso al servicio, seguridad, } \\
\text { tarifas e incluso qué instalaciones auxiliares se deben }\end{array}$ \\
\hline
\end{tabular}

(cc) EY-NC-ND 2018, Universitat Politècnica de València

Congreso IN-RED (2018) 


\begin{tabular}{|l|l|l|}
\hline LDO & $\begin{array}{l}\text { Lease-Develop- } \\
\text { Operate }\end{array}$ & $\begin{array}{l}\text { Equivalente al anterior pero en lugar de adquirir los activos, } \\
\text { el operador privado los arrienda a la Administración. La } \\
\text { inversión inicial es menor y el interés por la participación } \\
\text { del sector privado reside en las mejoras en eficiencia en la } \\
\text { prestación del servicio por su capacidad de gestión. }\end{array}$ \\
\hline WAA & $\begin{array}{l}\text { Wraparound } \\
\text { Addition }\end{array}$ & $\begin{array}{l}\text { Un operador privado financia y construye la ampliación de } \\
\text { una infraestructura pública ya existente. Posteriormente } \\
\text { gestiona y explota comercialmente ambas partes, bien por } \\
\text { un período establecido, o bien hasta que haya recuperado la } \\
\text { inversión inicial y una rentabilidad sobre la misma. }\end{array}$ \\
\hline
\end{tabular}

Fuente: Catalá Pérez, D. (2017)

Al finalizar la práctica, cada grupo pegó sus notas en orden en un folio (o los necesarios) y lo entregó al profesor con el nombre de los integrantes del equipo (Imagen 1).

\section{Resultados}

La propuesta: ¿es posible un sistema de colaboración público-privada contractual para el servicio de taxis?, fue resuelta por todos los grupos de manera positiva, buscando una solución al problema.

En primer lugar, identificaron el mapa de actores (Figura 1).

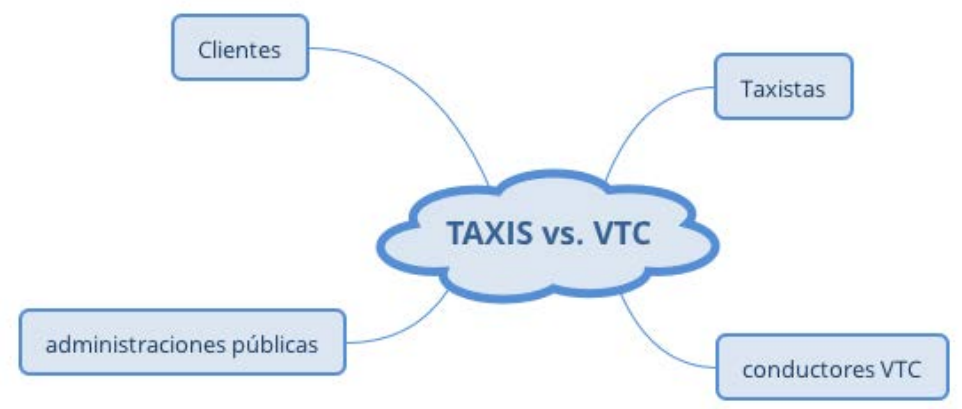

Fig. 1 Mapa de actores de la "guerra de los taxis".

Fuente: elaboración propia.

Desde el punto de vista de los usuarios-clientes, entre los aspectos positivos del uso de plataformas con conductores VTC (tipo Uber), se destacaron una aplicación sencilla de manejar, coches amplios y limpios y un precio total menor (aunque el mínimo suele ser mayor). En cuanto a los aspectos negativos, los principales son el tiempo de espera que suele ser mayor (y no se puede acudir a una parada) y una menor oferta (puesto que hay menos licencias VTC que de taxis).

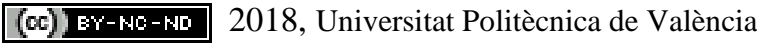

Congreso In-Red (2018) 
Desde el punto de vista de los taxistas, la queja principal es que las licencias VTC son más baratas que las licencias de taxis y en algunas ciudades no se cumplen ratios VTC/taxis, lo que satura la oferta de servicio. Sin embargo, los conductores VTC quieres hacerse un espacio que no acaban de encontrar en un sector que se supone liberalizado pero que todavía se rige por muchas normas que deberían revisarse.

Por último, el papel que juegan las administraciones públicas no es sencillo, puesto que hay muchos intereses en conflico, por un lado el de grandes empresas como Uber o Cabify, y por otro el del sector del taxi que es un fuerte lobby en España. En la actualidad no parece que vaya a haber muchos cambios normativos, pero la economía digital es un fenómeno imparable por lo que no es una situación que pueda sostenerse mucho tiempo.

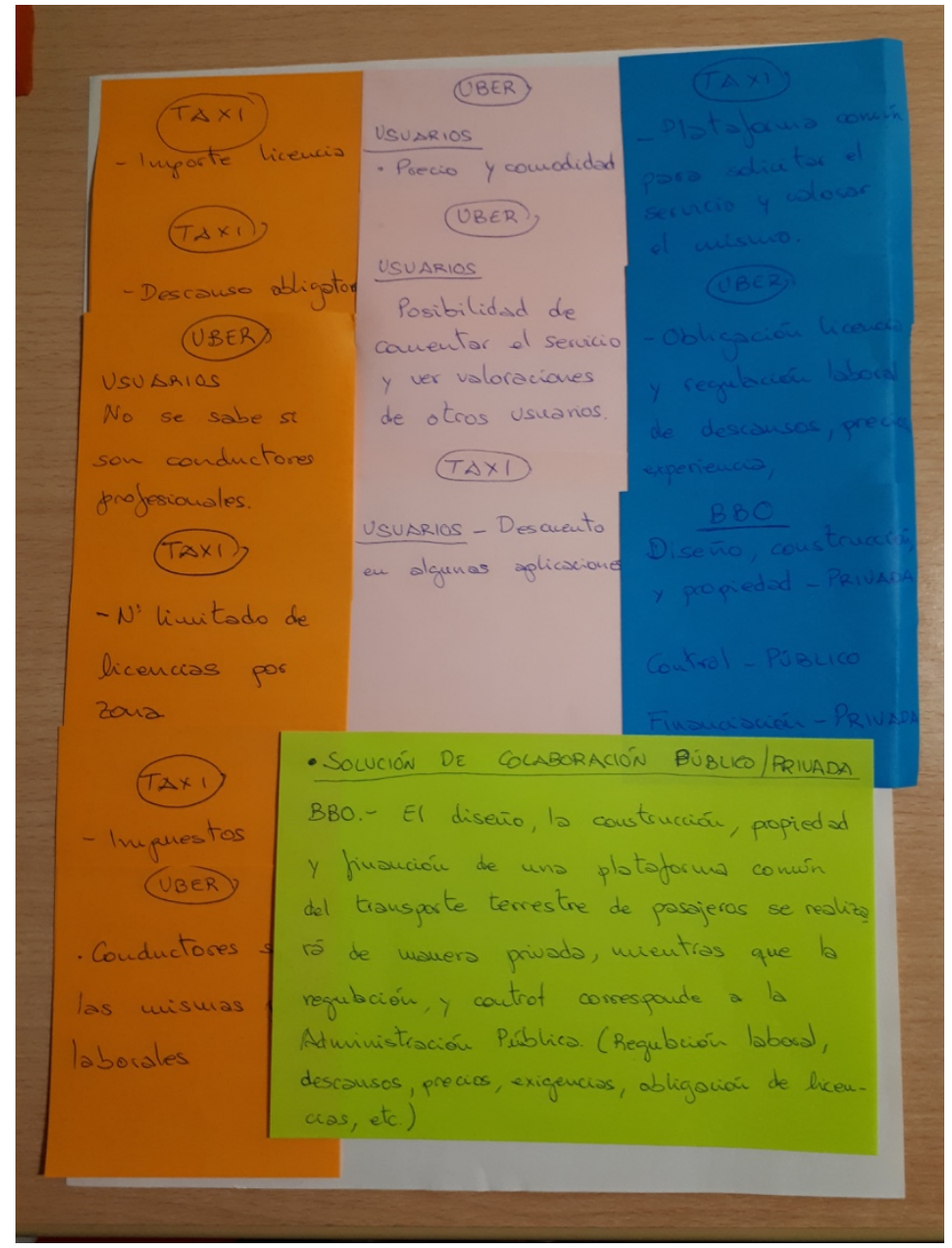

Imagen. 1 Resolución de la práctica por uno de los grupos, curso 2017-2018.

Fuente: elaboración propia 
En cuanto a las posible soluciones de CPP, de los 4 grupos, 3 se decantaron por la misma fórmula: BBO (Buy-Build-Operate), si bien el peso que le dio cada grupo al sector público o privado varió. En uno de los grupos, se le dio todo el "peso" del contrato a la administración pública, mientras que en los otros dos grupos el sector privado asume más riesgos, haciéndose cargo de la construcción y gestión de una plataforma digital y dejando únicamente a la administración pública la función de control y regulación. Solo el cuarto grupo escogió otra fórmula, la WAA (Wraparound Addition), transfiriendo mayores riesgos al sector privado pero dejando en manos de la administración el control y la regulación.

\section{Conclusiones}

Nos encontramos con un problema complejo que necesita una análisis sistémico para encontrar soluciones, con la participación de todos los grupos de interés en conflicto.

Se ha podido observar que, por un lado, hay poco control en el traspaso o adquisición de licencias en ambos casos, pero, por otro lado, se quiere mantener un modelo de negocio que no es sostenible en el escenario actual.

La práctica ha permitido a los alumnos del Grado en Gestión y Administración Pública aplicar unos conocimientos complejos, como son los de la colaboración público-privada, a una problema actual y que les es cercano, por lo que pueden interiorizar mejor esos conocimientos. Evidentemente, una práctica de 2 horas no puede llegar a unas conclusiones profundas del problema, pero se introduce el tema y se enseña una metodología que les puede servir en su futuro profesional a la hora de tomar decisiones en grupo.

Para ello se ha utilizado como técnica, desde un enfoque de stakeholders o grupos de interés, la toma de decisiones en equipo mediante el uso de notas móviles.

\section{Agradecimientos}

Este trabajo parte del proyecto de investigación Prometeo en el que participa la profesora María De Miguel, “La regulación de la transformación digital y la economía colaborativa”, y que es dirigido por el profesor Lorenzo Cotino Hueso (PROMETEO/2017/064, Generalitat Valenciana).

Así mismo, ha formado parte del Proyecto de Innovación y Mejora Educativa (PIME), "La resolución de problemas a través del diseño creativo y la visualización colaborativa” de la UPV, coordinado por la profesora V. Santamarina.

\section{Referencias}

CATALÁ-PÉREZ, D. (2017). “La colaboración público-privada” en De-Miguel-Molina, M.; Bañón Gomis, A.J.; Catalá-Pérez, D. Management para las Administraciones públicas. Valencia: Editorial Universitat Politècnica de València, pp. 131-158. 
Comisión Europea. Comunicación de la Comisión al Parlamento Europeo, al Consejo, al Comité Económico y Social Europeo y al Comité de las Regiones. Una Agenda Europea para la economía colaborativa. Comisión Europea, 2 de junio de 2016, COM(2016) 356 final, 1-18.

DE-MIGUEL-MOLINA, M.; DE MIGUEL MOLINA, B.; SANTAMARINA CAMPOS, V.; CARABAL-MONTAGUD, A. (2017a). "Problem-solving in group using mobile notes" en 11th International Technology, Education and Development Conference (INTED 2017, Valencia). Valencia: IATED Digital Library. 3825-3830.

DE-MIGUEL-MOLINA, M.; BAÑÓN GOMIS, A.J.; CATALÁ-PÉREZ, D. (2017b). Management para las Administraciones públicas. Valencia : Editorial Universitat Politècnica de València.

DOMENECH PASCUAL, G. (2015). "La Regulación de la Economía Colaborativa. El caso «Uber contra el taxi»” en Revista CEFlegal, n. 175-176, pp. 61-104.

DOMÍNGUEZ DEL VALLE, E. (2017) "En contra: Mejor un taxi público que uno privatizado" en El País, 11 de mayo, $<$ https://elpais.com/elpais/2017/05/10/opinion/1494436161_707931.html> [Consulta: 4 de febrero de 2018]

ESTEVE, J. (2016) "Uber, Cabify o Car2Go: probamos las alternativas al taxi. ¿Cuál es mejor?” en El Confidencial, 5 de abril, <http://www.elconfidencial.com/tecnologia/201604-05/uber-taxi-cabify-car2go-alternativas-transporte-madrid_1178533/> [Consulta: 4 de febrero de 2018]

FERNÁNDEZ, D. (2017) "El TS inflama la guerra del taxi en Madrid con 3.000 nuevos permisos de Uber y Cabify" en El Confidencial, 15 de febrero, $<$ http://www.elconfidencial.com/espana/madrid/2017-02-15/supremo-taxi-madridlicencias-vtc_1331985/> [Consulta: 4 de febrero de 2018]

HERCE, J.A (2017) “ A favor: la movilidad en las ciudades inteligentes” en El País, 12 de mayo, <https://elpais.com/elpais/2017/05/09/opinion/1494349480_105972.html?rel=mas> [Consulta: 4 de febrero de 2018]

MYTAXI. Acerca mytaxi. <https://es.mytaxi.com/taxistas.html > [Consulta: 4 de febrero de 2018]

OTERO, C. (2017) "Por qué Cabify, Uber y similares enfadan tanto a los taxistas" en AS (Betech), 30 de mayo, $<$ https://as.com/betech/2017/05/30/portada/1496154650_719396.html> [Consulta: 4 de febrero de 2018]

TSJUE. Sentencia del Tribunal de Justicia (Gran Sala) Procedimiento prejudicial Artículo 56 TFUE - Artículo 58 TFUE, apartado 1 - Servicios en el ámbito de los transportes - Directiva 2006/123/CE - Servicios en el mercado interior - Directiva 2000/31/CE - Directiva 98/34/CE - Servicios de la sociedad de la información Servicio de intermediación que permite, mediante una aplicación para teléfonos inteligentes, conectar a cambio de una remuneración a conductores no profesionales que utilizan su propio vehículo con personas que desean realizar desplazamientos urbanos Exigencia de una autorización». InfoCuria, 20 de diciembre de 2017, Asunto C-434/15, 112. 